\section{Den sekundären Hodenhochstand gibt es wirklich}

$D^{i}$ ie sekundäre Aszension des Hodens kommt offenbar sehr viel häufiger vor als bislang angenommen. Dass es so etwas wie einen erworbenen Hodenhochstand gibt, hätte sie vor 20 Jahren kategorisch bestritten, meinte PD Dr. med. Annette Schröder vom Krankenhaus Barmherzige Brüder in Regensburg. Dabei beschrieben bereits im Jahre 1966 Wissenschaftler in einer dänischen Studie bei der Beobachtung der spontanen Entwicklung der Hodenlage von 4.300 Jungen die Aszension nach bereits stattgehabtem Deszensus mit einer Rate von 1,6\% [Villumsen AL, et al. Arch Dis Child. 1966;41:198-200].

Zehn Jahre später gab es einen Report über zwei Familien, in denen acht der neun Söhne eine Aszension entwickelten. In größeren Abständen folgten weitere Berichte, stets begleitet von Zweifeln.
Im Jahre 2003 erschien eine niederländische Studie, wonach bei 261 Jungen, die zur Operation eines Hodenhochstands kamen, aufgrund von Befragungen ermittelt worden war, dass bei nicht weniger als $73 \%$ von ihnen der Hoden bereits mindestens einmal im Skrotum gewesen sei [Hack WW, et al. BJU Int. 2003;92:293-6]. Schröder und ihre Kollegen haben diese Untersuchung zwischen Mai 2015 und August 2016 an ihren eigenen Patienten in Regensburg wiederholt. Bei 175 Jungen, die zur Operation kamen, waren die Anamnese bezüglich der früheren Hodenlage erhoben und zusätzlich U-Hefte und alte Krankenakten gesichtet worden. 94 hatten einen primären Hodenhochstand und 81 (46\%) hatten eine Aszension. Von den Patienten mit Aszension hatte wiederum die Hälfte zu- vor einen Pendelhoden. Dies scheine neben der positiven Familienanamnese, einer Hydrozele sowie der späten Deszension des Hodens nach der Geburt ein Risikofaktor zu sein, fasste Schröder zusammen.

Sie wies darauf hin, dass die Fertilität dieser Patienten später ähnlich eingeschränkt sei wie bei primärem Hodenhochstand, wobei einseitig betroffene Jungen mit geringen Einschränkungen rechnen müssen, im Unterschied $\mathrm{zu}$ beidseitig betroffenen.

Schröder forderte, die Hodenlage bis zur Pubertät regelhaft zu kontrollieren, etwa zu den U-Terminen. Bei hochskrotal liegenden Hoden sollte kurzfristig nachkontrolliert werden. Steht die Diagnose eines aszendierten Hodens fest, müsse so zügig wie möglich operiert werden.

Dr. Thomas Meißner

Forumssitzung "Gibt es einen erworbenen Hodenhochstand (aquired testis?)", A. Schröder, DGU-Kongress, Leipzig, 28. September 2016

\section{Online-Entscheidungshilfe Prostatakrebs kommt bei Patienten und Ärzten gut an}

$\mathrm{D}$ ie Online-Entscheidungshilfe Prostatakrebs ist gut angelaufen. Erste Nutzerdaten bestätigen eine hohe $\mathrm{Zu}$ friedenheit bei den Patienten. Am 1. Juni 2016 war die Online-Entscheidungshilfe für Männer mit lokalisierten Prostatakarzinomen an den Start gegangen - abrufbar unter www.entscheidungshilfeprostatakrebs.info. Sie war auf Basis der S3-Leitlinie sowie unter dem Dach der PatientenAkademie der Deutschen Urologen entwickelt worden.

Über Videosequenzen kann sich der Patient in Ruhe zuhause und gemeinsam mit seinen Angehörigen über seine Krankheit informieren. Ein Filmarzt ein für das Projekt engagierter Schauspieler - leitet den Patienten individualisiert durch das Online-Programm. Möglich wird das mithilfe von acht Angaben, die der Patient in das Programm eingibt. Die Angaben erfolgen beispielsweise zum initialen PSA-Wert, GleasonScore oder klinischem TNM-Stadium. Das Konzentrat der vom Patienten ein- gegebenen Informationen erhält der Urologe in Form eines automatisch generierten, zusammenfassenden Protokolls im DIN-A4-Format. Auf diese Weise werden sowohl der Patient als auch der Arzt in die Lage versetzt, informiert und zeiteffektiv gemeinsam Entscheidungen über das weitere Vorgehen zu treffen.

„Von Juni bis August 2016 nutzten 319 Patienten die Entscheidungshilfe“, erklärte der Initiator und gleichzeitig Leiter des Projekts PD Dr. med. Johannes Huber vom Universitätsklinikum Carl Gustav Carus in Dresden. Es seien monatlich steigende Teilnehmerzahlen $\mathrm{zu}$ beobachten. $87 \%$ der Patienten waren mit dem internetbasierten Werkzeug zufrieden oder sogar sehr zufrieden. Auch von den teilnehmenden Urologen gebe es durchweg eine positive Rückmeldung, betonte Huber.

Die bislang ausgewerteten Teilnehmer waren durchschnittlich 66 Jahre alt, knapp 69 \% hatten alle Fragebögen voll- ständig beantwortet. Bei drei Viertel der Patienten bestand ein niedriges oder mittleres onkologisches Risiko, bei $26 \%$ ein hohes Risiko. Im psychoonkologischen Screening fanden sich bei $29 \%$ der Patienten Hinweise auf eine Depression und bei knapp $30 \%$ auf eine Angstproblematik. „Die psychische Belastung erschien bei $34,2 \%$ auffällig hoch“, resümierte Huber.

$58 \%$ der Patienten nutzten das Programm gemeinsam mit ihrem Partner. Dabei zeigte sich das Online-Medium im Vergleich zu gedruckten Aufklärungsmaterialien als vorteilhafter.

Sobald sich die Online-Entscheidungshilfe etabliert hat, sollen die Effekte in einer randomisierten Evaluationsstudie gemessen werden. Zusätzlich würden Versorgungsdaten generiert, die in ihrer Tiefe einzigartig wären, wie Huber sagte.

Dr. Thomas Meißner

DGU-Pressekonferenz "Wie kommt die ,Entscheidungshilfe Prostatakrebs' bei Patienten und Urologen an? Erste Evaluationsergebnisse zum neuen Beratungsportal der PatientenAkademie", J. Huber, DGU-Kongress, Leipzig, 29. September 2016 